\title{
Singularity parameter determination in adhesively bonded lap joints for use in failure criteria.
}

\author{
A. Barroso, V. Mantič, F. París. \\ (abc@esi.us.es,mantic@esi.us.es,paris@esi.us.es) \\ Group of Elasticity and Strength of Materials, University of Seville, Spain.
}

\begin{abstract}
A procedure for the singularity characterization of anisotropic multimaterial corners which typically appear in adhesively bonded lap joints between metals and composites is presented and implemented in the present work. The characterization in terms of characteristic exponents (stress singularities), characteristic functions and generalized stress intensity factors allows the definition of an oriented test program in order to analyze the suitability of a singularity based failure criterion for structures of this type.
\end{abstract}

Keywords: A) Adhesive joints, C) Interfacial strength, C) Failure criterion, C) Stress singularity.

\section{INTRODUCTION}

Adhesively bonded joints between metallic sheets and composite laminas generate critical points, where the Linear Theory of Elasticity predicts unbounded stresses. These singularity stress fields are due to abrupt changes in geometry and/or abrupt changes in the elastic properties between the different materials, Sinclair [1]. In the particular case of adhesively bonded single (or double) lap joints between an aluminium sheet and a CFRP laminate, these critical points can be easily identified in Fig. 1 a.

\section{Fig 1}

With a polar coordinate system $(r, \theta)$ located at the multimaterial corner tip (Fig. 1b), where $\theta_{1-1}$ and $\theta_{1}$ are the angles of the $i$-th material wedge ( $N$ wedges), the asymptotic singularity stress field in the vicinity of the corner can be assumed to have the following series expansion structure with separation of variables (some particular corner configurations, which will not be considered here, do not admit the separation of variables, see for example Joseph and Zhang [2]). 


$$
\begin{aligned}
& \sigma_{i j}(r, \theta)=\sum_{k=1}^{n} \frac{K_{k}}{(2 \pi r)^{1-\lambda_{k}}} f_{i j k}(\theta), \quad i, j=r, \theta, \\
& u_{i}(r, \theta)=\sum_{k=1}^{n} \frac{K_{k} r^{\lambda_{k}}}{(2 \pi)^{1-\lambda_{k}}} g_{i k}(\theta), \quad i, j=r, \theta,
\end{aligned}
$$

where $n$ is the number of terms considered in the series expansion, $\lambda_{k}$ are the characteristic exponents, leading to singular terms when $0<\lambda_{k}<1$ and regular terms if $\lambda_{k} \geq 1, f_{i j k}(\theta)$ and $g_{i k}(\theta)$ are the characteristic functions: smooth functions inside each material wedge which contain the angular dependency of the stress and displacement components respectively associated to the singularity mode $\lambda_{k}$ and $K_{k}$ are the Generalized Stress Intensity Factors (GSIFs) which represent the weight of each term in the asymptotic series expansion.

The characterization of the singular stress field is fundamental if the initiation of failure (an initial crack or localized damage whose propagation implies the complete failure of the joint) can be assumed to occur due to that singular stress field.

In the same way as Linear Elastic Fracture Mechanics (LEFM) accurately predicts the propagation of a crack when the $\operatorname{SIF}\left(K_{k}\right)$ reaches a certain allowable value (the fracture toughness of the material), it seems reasonable to consider the possibility of a GSIF based failure criterion (see Gradin \& Groth [3], Groth [4], Hattori [5], Reedy [6], Reedy \& Guess [7], Akisanya [8], Yang \& Munz [9], Lefebvre \& Dillard [10], Ishii et al [11], Wang \& Rose [12], Dunn et al. [13], Penado [14], Lazzarin et al [15], Leguillon \& Yosibash [16] and Quaresimin \& Ricotta [17] among others). To analyze the suitability of such an approach using the concepts of the LEFM, it is necessary to have the tools to evaluate $\lambda_{k}, f_{i j k}(\theta), g_{i k}(\theta)$ and $K_{k}$. Only after this careful characterization can experimental tests be carried out in an oriented way, changing in a controlled manner the values of the singularity parameters, in order to observe their influence (if it occurs) in a failure initiation.

In successive sections we will introduce the analytical procedure to determine the characteristic exponents and characteristic functions (Section 2), and the numerical models (in BEM) as well as the implemented procedure based on a least squares determination of the GSIFs (Section 3). The accuracy of the method is checked with some benchmark problems, and some examples regarding the characterization of adhesively bonded lap joints between metal and composites are presented (Section 4). With these tools, a preliminary experimental test program can be specifically designed to investigate the influence of the singularity parameters on failure initiation.

\section{CHARACTERIZATION OF THE SINGULARITY MODES}

The characteristic exponents $\lambda_{k}$ and the characteristic functions $f_{i j k}(\theta)$ and $g_{i k}(\theta)$ only depend on material elastic properties, local geometry and local boundary conditions. 
Considering homogeneous linear elastic materials, perfect adhesion between the material wedges, homogeneous boundary conditions (at external faces) and plane states (plane stress, plane strain or generalized plane strain), at the present moment there is enough information and efficient analytical and computational tools for the evaluation of accurate values of $\lambda_{k}$, see Dempsey and Sinclair [18,19], Pageau et al. [20], Ting [21], Mantič et al. [22] and Poonsawat et al. [23,24] among others. In this work a semianalytical tool, developed by the authors, Barroso et al. [25], is employed.

Some of the main characteristics and improvements of the present tool in comparison with the previous works are explained briefly in the following.

All linear elastic material behaviour laws (from isotropic to fully anisotropic) can be included simultaneously in the analysis. Depending on the approach, it has often been observed in previous works that only material behaviour laws of one kind (only isotropic, or only orthotropic) have been considered in the analysis. When considering anisotropic materials and using the powerful and elegant Lekhnitskii-Eshelby-Stroh formalism for anisotropic elasticity: Lekhnitskii [26], Eshelby et al. [27] and Stroh $[28,29]$, or simply, Stroh formalism in what follows, these limitations were usually associated to the fact that isotropic materials are, in the framework of the Stroh formalism, mathematically degenerate materials and the formalism becomes much more cumbersome, see Ting and Hwu [30] and Wang and Ting [31].

A particular effort has been focused on obtaining explicit expressions of the Stroh matrices ( $\mathbf{A}$ and $\mathbf{B}$ ) for transversely isotropic materials, a material behaviour which can be assumed to represent the unidirectional long fibre composite laminas. These expressions have also been properly orthogonalized and normalized for the powerful orthogonality and closure relationships of the formalism to be fulfilled. These expressions, Barroso et al. [32], will help to develop further application of the Stroh formalism to transversely isotropic materials. It is of major importance to notice that mathematically degenerate cases can appear when considering transversely isotropic materials (see Tanuma [33]) irrespective of the value of the elastic constants of the material and only depending on the relative orientation of the material, a fact that has also been considered in the program implementation.

All typical orthogonal homogeneous boundary conditions can be imposed at the external faces of the multimaterial corner (see Table 1), and closed corners (called also cross points, all materials bonded, no external faces) can also be analyzed. The matrices in Table 1 are defined in terms of the outward normal (n) and tangential vectors $\left(\mathbf{s}_{\mathrm{r}}, \mathbf{s}_{3}\right)$ (see Fig. 2 ) at the external faces $\left(\theta_{0}, \theta_{\mathrm{N}}\right)$ with $\mathbf{0}$ and $\mathbf{I}$ being respectively the null and identity matrices.

Fig 2

Table 1 
Following an original idea by Ting [21], the concept of transfer matrix has been further applied to degenerate and extraordinary degenerate materials, so that independently of the number and type of materials in the corner, the characteristic equation from which the characteristic exponents are obtained is derived in a simple manner, always given by the vanishing condition of a $3 \times 3$ determinant in the case of open corners (or $6 \times 6$ determinant for closed corners). The characteristic equation written as:

$$
\left|\hat{\mathbf{K}}_{N}^{(2)}(\lambda)\right|=\mathbf{0},
$$

with

$$
\begin{aligned}
\hat{\mathbf{K}}_{N}^{(2)} & =\mathbf{D}_{u}\left(\theta_{N}\right) \mathbf{K}_{N}^{(1)} \mathbf{D}_{\varphi}^{T}\left(\theta_{0}\right)+\mathbf{D}_{u}\left(\theta_{N}\right) \mathbf{K}_{N}^{(2)} \mathbf{D}_{u}^{T}\left(\theta_{0}\right)+ \\
& +\mathbf{D}_{\varphi}\left(\theta_{N}\right) \mathbf{K}_{N}^{(3)} \mathbf{D}_{\varphi}^{T}\left(\theta_{0}\right)+\mathbf{D}_{\varphi}\left(\theta_{N}\right) \mathbf{K}_{N}^{(4)} \mathbf{D}_{u}^{T}\left(\theta_{0}\right)
\end{aligned}
$$

where $\mathbf{D}_{u}\left(\theta_{a}^{e}\right)$ and $\mathbf{D}_{\varphi}\left(\theta_{a}^{e}\right)$ are defined in Table 1 and $\mathbf{K}_{N}^{(i)}$ are the submatrices of $\mathbf{K}_{N}$ which is the transfer matrix of the entire corner and is obtained as the product of the transfer matrices of the material wedges $\mathbf{E}_{N}$ ( $N$ being the number of material wedges in the corner):

$$
\mathbf{K}_{N}=\mathbf{E}_{N} \cdot \mathbf{E}_{N-1} \cdot \ldots \cdot \mathbf{E}_{2} \cdot \mathbf{E}_{1}
$$

The structure of a transfer matrix $\mathbf{E}_{i}$ is detailed in Ting [21] for non-degenerate cases and in Barroso et al. [25] for all cases of mathematical degeneracy.

The characteristic functions $f_{i j k}(\theta), g_{i k}(\theta)$ associated to $\lambda_{k}$ can also be numerically evaluated, always bearing in mind that GSIFs have to be defined together with these characteristic functions for the stress field to be completely and coherently defined, see Yosibash \& Szabo [34] and also Pageau et al. [35].

In short, the tool presented in this section permits an analytical knowledge of the characteristic exponents and functions, which will be considered to be known in the next section.

\section{GENERALIZED STRES INTENSITY FACTORS}

In the evaluation of the GSIFs, the global geometry and far field loading are now necessary, thus numerical models or experimental tests (e.g. using photoelasticity) have to be used. Additionally, in most cases, a post processing of the data is necessary, the accuracy of the results being finally dependent on the "quality" of both processes. For example, due to the asymptotic character of the elastic representation in (1), GSIFs obtained from the solution far from the corner tip can not be representative of the real elastic solution, while GSIFs obtained extremely near the corner tip can be affected by numerical errors associated to the model discretization. 
There exist techniques which overcome these difficulties by means of path-independent integrals evaluated in the neighbourhood of the corners, see for example Qian and Akisanya [36], Wu [37], Banks-Sills and Sherer [38] and Cisilino \& Ortiz [39] among others. Further information on these different techniques can be found in Helsing and Jonsson [40] and Sinclair [1].

Unlike the typically high accuracy found in the literature regarding the evaluation of characteristic exponents, the accuracy in the evaluation of GSIFs is substantially worse. More reliable results for benchmark problems are needed which could be used as reference values in new evaluation methods. Some interesting comments regarding the validity of published numerical results in the literature can be found in Helsing and Jonsson [41].

In the present work, with the previous knowledge of accurate values of the characteristic exponents and characteristic functions, a simple procedure has been implemented based on a least squares method in terms of displacements and/or stresses. The numerical models have been performed using a BEM code developed by Graciani [42]. BEM models are more suitable (when compared with FEM models) due to their higher accuracy in solving problems involving singular stresses.

Extending the approach developed by Munz and Yang [43] using FEM, the present work uses a least squares method to evaluate the GSIF values. An error function $J$ (5a) is defined as summing squares of differences between the displacements, $J_{u}(5 \mathrm{~b})$, and/or tractions, $J_{t}(5 \mathrm{c})$, obtained from the numerical model $\left(u^{B E M}\right.$ and $\left.T^{B E M}\right)$ and the corresponding values obtained from the asymptotic series expansion in (1) ( $u^{\text {series }}$ and $\left.T^{\text {series }}\right)$. The function $J$ depends on the values of $a$ and $b$, the usual choices being $(a, b)=(0,1),(a, b)=(1,0)$ or $(a, b)=\left(l^{-2}, \sigma^{-2}\right)$ where $l$ and $\sigma$ are some characteristic length and stress values.

$$
\begin{gathered}
J\left(K_{1}, \ldots, K_{n}, K_{0 q}\right)=a J_{u}+b J_{t} \quad(a, b \geq 0) \\
J_{u}=\sum_{\alpha=1}^{A} \sum_{j=0}^{B} \sum_{n=1}^{M}\left[u_{\alpha}^{B E M}\left(r_{n}, \theta_{j}\right)-u_{\alpha}^{\text {series }}\left(r_{n}, \theta_{j}, K_{1}, \ldots, K_{n}, K_{0 q}\right)\right]^{2} \\
J_{t}=\sum_{\alpha=1}^{A} \sum_{j=0}^{B} \sum_{n=1}^{M}\left[T_{\alpha}^{B E M}\left(r_{n}, \theta_{j}\right)-T_{\alpha}^{\text {series }}\left(r_{n}, \theta_{j}, K_{1}, \ldots, K_{n}\right)\right]^{2}
\end{gathered}
$$

The only unknowns in $J$ are the GSIFs, $K_{k}(k=1, \ldots, n)$ and the rigid body translations $K_{0 q}$ (if necessary). In the $J$ expression three summatories are considered: $A$ represents the number of displacement components ( $A=1$ for $u_{r}, u_{\theta}$ or $u_{z}$ only, $A=2$ for $u_{r}$ and $u_{\theta}, u_{r}$ and $u_{z}$ or $u_{\theta}$ and $u_{z}$ and $A=3$ for $u_{r}, u_{\theta}$ and $u_{z}$ ) $B$ defines the number of edges used in the evaluation of the error (with $N$ material wedges, there exist $N+1$ edges where the BEM model gives direct results, without internal points) and finally $M$ indicates the number of nodes along an edge used in the evaluation of $J$. An approximation for $K_{k}$ (and $K_{0 q}$ ) is obtained from the solution of the following linear equation system: 


$$
\frac{\partial J\left(K_{1}, \ldots, K_{n}\right)}{\partial K_{k}}=0,(k=1, \ldots, n) \quad \frac{\partial J\left(K_{1}, \ldots, K_{n}, K_{0 q}\right)}{\partial K_{0 q}}=0, \quad(q=x, y, z)
$$

The authors have successfully checked the procedure, defined by (5) and (6), using displacements and stresses as well. In the next section the benchmark problems present corners with free-free boundary conditions, where only displacements have been used in the evaluation of the GSIFs, and, for comparison purposes, the rest of the problems analyzed have been evaluated using only displacements $(a, b)=(1,0)$.

\section{BENCHMARK PROBLEMS AND APPLICATIONS}

To analyze the accuracy of the implemented method, two simple problems have been selected, a $90^{\circ}$ notch with tensile loadings and an isotropic free-free bimaterial corner.

Although the method is independent of the complexity of the problem (number and type of materials, boundary conditions, etc.), it seemed reasonable to check it at least in these two problems. Nevertheless, the lack of benchmark problems in the literature to check with is evident. As mentioned before, the values of the GSIFs depend on of the definition of the characteristic functions (Yosibash and Szabo [34]), and many authors do not present both of them, the difficulty of such comparisons also being due to the lack of uniformity and information in the normalization/standardization of the presented results as indicated by Pageau et al [35].

\subsection{Single-edge $90^{\circ}$ notched specimen subjected to tensile loading.}

This simple problem (Fig. 3) has been analyzed with $a / w=0.5$ and $h / w=1$, with $a=5 \mathrm{~cm}$. The BEM model has 709 nodes and 709 linear elements, with a size along the outer edges of $0.1 \mathrm{~cm}$. Along the edges converging at the corner the element adjacent to the tip has a size of $10^{-7} \mathrm{~cm}$, growing with a factor of 1.5 until a size of $0.1 \mathrm{~cm}$ is reached, with a total of 100 elements along these faces.

\section{Fig 3}

This symmetric load configuration, which generates a mode I (opening) in the neighbourhood of the corner, has been analyzed in detail by Helsing and Jonsson [40] from where the reference value of $K_{\mathrm{I}}=4.295886967699$ has been taken.

Using $u_{r}$ and $u_{\theta}(A=2)$ and both edges $(N=2)$ for the evaluation of the error $J$ in $(5)$, Fig. 4 represents the absolute value of the relative error (up to 5\%) between the obtained solution and the reference considering: a) three and b) seven terms in the asymptotic series expansion in (1). It is important to notice that horizontal axes do not represent distances to the notch tip but only node numbers, and although element length far from the tip is constant, element lengths near to the notch tip change gradually. 
All possible combinations resulting from a consecutive group of nodes have been considered, the right hand side axis being the initial node number and the left hand side axis the final node number (the bottom half part of plots in Fig. 4 then being meaningless). It is clear that major errors are associated to:

- A small group of nodes far away from the notch tip (the peak at the left hand side of Fig. 4) where the more regular terms of the asymptotic expansion and also possibly the external boundary obviously affect the solution.

- A small group of nodes very close to the notch tip (the peak at the right hand side of Fig. 4), where numerical errors associated to the discretization affect the solution.

\section{Fig 4}

The more terms of the asymptotic expansion are considered in the analysis, the lower the errors associated to the far group of nodes are achieved. The previous consideration is clear when comparing Fig. 4a) and 4b). In both cases, it can be observed that, from an engineering point of view, for most of the "reasonable" group of nodes (avoiding small groups of nodes far away and very close to the notch tip) results are accurate enough $(<3 \%)$.

For the same configuration, and using a path-independent technique, Ortiz et al. [44], the errors obtained are always below $1 \%$.

\subsection{Isotropic bimaterial corner.}

The tools developed in Sections 2 and 3 have also been used to analyze the bimaterial corner in Fig. 5, (with $h=1$ ) from Qian and Akisanya [36].

\section{Fig 5}

The reference solution reported by Qian and Akisanya was obtained for Dundurs parameters given by $\alpha=0.8$ and $\beta=0.2$ which indicate that the material in the top part of Fig. 5 is about ten times stiffer than the material in the bottom part.

Using a FEM model and path-independent integrals, finally transformed into domain integrals, Qian and Akisanya obtained for the first three terms (only the first one being singular) the following values for $\lambda_{k}$ and $a_{k}\left(a_{k}\right.$ being a dimensionless $K_{k}$ ): $\lambda_{1}=0.6747$ ( $\left.a_{1}=0.6301\right), \lambda_{2}=1.1637\left(a_{2}=-0.3671\right)$ and $\lambda_{3}=1.5938\left(a_{2}=0.5443\right)$.

The BEM model used in the present work has linear elements with a size of $0.025 \mathrm{~h}$ far away from the corner and $10^{-8} h$ at the corner tip. The characteristic exponents obtained using the semi-analytical tool developed in Barroso et al. [25] were $\lambda_{l}=0.673473$, $\lambda_{2}=1.167477, \lambda_{3}=1.589147$, very similar to those obtained by Qian and Akisanya. The 
evaluation of the GSIFs was also performed using (5) and (6) with $u_{r}$ and $u_{\theta}(A=2)$, the three edges which converge at the corner $(N=3)$ and an average value from the flattest part of reasonable groups of nodes (the central top part of the corresponding representation, similar to Fig 4b), avoiding far and close nodes to the corner tip.

Considering the first three terms of the asymptotic representation, the obtained value was $a_{1}=0.673688$, which differs $6.92 \%$ from the result by Qian and Akisanya. Ortiz et al. [44] for the same problem and a path-independent technique reported an average value of $a_{1}=0.67829$, which differs $7.65 \%$ from Qian and Akisanya and $0.68 \%$ from the present results.

The good agreement reported by Qian and Akisanya (using FEM) in the range $10^{-4} \mathrm{~h}$ and $10^{-1} h$ is improved by the present results, see Fig. 6 (for internal points at $r=0.1 \mathrm{~h}$ ) with a BEM model. The range used with the least squares method in the present work is $5.818585 \cdot 10^{-6} h<r<0.6224868 h$, which leads to a closer agreement between numerical and analytical results.

\section{Fig 6}

Once the tool has been demonstrated to be accurate in these particular problems, it will be used to characterize the multimaterial corners which typically appear in adhesively bonded joints between metals and composites.

Note that only stresses $(a, b)=(0,1)$ have also been used in a least squares expression $(5)$, instead of only displacements $(a, b)=(1,0)$, obtaining similar results with differences below $2 \%$. In this particular case, as the corner faces are stress free boundaries, only the common interface (at $0^{\circ}$ ) could be used to evaluate $K_{\mathrm{k}}$ by the least squares using

$\sigma_{\theta \theta}$.

\subsection{Adhesively bonded joint between a metal and a composite.}

Different test configurations can be specifically designed to analyze the suitability of a singularity based failure initiation criterion by means of previous knowledge of the singularity parameters. For example, as GSIF dimensions depend on the associated characteristic exponents $\lambda_{k}$, only GSIFs associated to the same value of $\lambda_{k}$ can be strictly compared. Accordingly, the following four different joint configurations (Fig. 7, where $\mathrm{L}_{\mathrm{o}}$ is the overlap length and the numbers between brackets at the caption are the thicknesses in $\mathrm{mm}$ ) with the same local corner configuration can be analyzed (only the overlap zone is detailed) in which the inner adherent is the metallic sheet and the first outer material of the composite adherent is a $0^{\circ}$ layer:

To show the capabilities of the previously introduced tools, the numerical analysis of configuration in Fig. 7a) is presented. The bonded double-lap joint is loaded in shear by means of a tensile force (see Fig. 8, where the units of the axes are the dimensions of the model in $\mathrm{mm}$ ). The symmetry of the double-lap joint configuration is used to model 
half of the geometry. Both the undeformed and the deformed geometry (x20) are shown in Fig. 8. Linear elements have been used and a progressive refinement towards the inner corner has been applied up to a final size of $10^{-8} \mathrm{~mm}$ at the corner tip, a total of 1484 nodes having been used in the BEM model.

\section{Fig 7}

The effect of curing residual stresses due to the polymerization temperature of the adhesive $\left(115^{\circ} \mathrm{C}\right)$ and the different thermal expansion coefficients (CFRP, adhesive and aluminium) has also been taken into account in the BEM model. The thermomechanical properties of the three materials used in the analysis are respectively: CFRP $\left(x_{1}\right.$ being the fiber direction, $E_{11}=141.3 \mathrm{GPa}, E_{22}=E_{33}=9.58 \mathrm{GPa}$ $v_{12}=v_{13}=0.3, v_{23}=0.32, G_{12}=5.0 \mathrm{GPa}, \alpha_{1}=-1 \cdot 10^{-6}{ }^{\circ} \mathrm{C}^{-1}, \alpha_{2}=\alpha_{3}=26 \cdot 10^{-6}{ }^{\circ} \mathrm{C}^{-1}$ ), aluminium $\left(E=68.67 \mathrm{GPa}, \nu=0.33, \alpha=24.5 \cdot 10^{-6}{ }^{\circ} \mathrm{C}^{-1}\right)$, epoxy adhesive $\left(3.0 \mathrm{GPa}, \nu=0.35, \alpha=45 \cdot 10^{-6}\right.$ $\left.{ }^{\circ} \mathrm{C}^{-1}\right)$. The applied tensile load is $400 \mathrm{~N}$ ( $200 \mathrm{~N}$ at each laminate at the right hand side).

Fig 8

Using the semi-analytical tool previously introduced (Barroso et al. [25]) the characteristic exponents $\left(\lambda_{k}\right)$ and functions $(f(\theta)$ and $g(\theta))$ have been obtained. The GSIFs associated to both mechanical and thermal loads have been evaluated using expressions (5) and (6) once the BEM model has been solved.

Results for the inner closed corner (corner "b" in Fig. 8 with $90^{\circ}$ wedge of $\left[0^{\circ}\right]$ CFRP and $270^{\circ}$ wedge of adhesive) and the outer corner at the left hand side of the adhesive spew fillet (corner "a" in Fig. 8 with $180^{\circ}$ aluminium wedge and $23^{\circ}$ adhesive wedge) are shown in Table $2\left(K_{\mathrm{g}}\right.$ associated to the characteristic exponent $\lambda_{\mathrm{k}}=1$ representing the rigid body rotation).

Table 2

The values of $K_{k}$ are standardized following Pageau et al. [35] in such a way that $\left.\sigma_{\theta}\right|_{\theta=0^{\circ}}=K /(2 \pi r)^{1-\lambda}$. In the particular case of the closed corner, and due to the low thickness of the adhesive layer $(\approx 0.1 \mathrm{~mm})$, the least squares adjustment has been performed between $r=10^{-6}$ and $r=0.03 \mathrm{~mm}$ (measured from the corner tip) to avoid the nearest nodes to the corner tip (between $10^{-8}$ and $10^{-6} \mathrm{~mm}$ ) and those which could be far away from the asymptotic representation ( $>1 / 3$ of the adhesive thickness).

The results for $r=0.0194 \mathrm{~mm}$ are plotted in Fig. 9, where circles are the BEM solution (at internal points) and the angle is measured from the horizontal interface between the CFRP laminate and the adhesive. Separate contributions of each term $\left(K_{1}, K_{2}\right.$ and $\left.K_{3}\right)$ are plotted separately, together with results considering two and three terms.

Fig 9 
It can be seen in Fig. 9 that three terms (the two singular and the first regular terms) are enough to accurately fit BEM results. It also has to be pointed out that the $K_{\mathrm{k}}$ used for this representation have been obtained from the nodes at the interfaces and the comparison performed in Fig. 9 is with internal points not used for the least squares adjustment, which is an additional verification of the correctness and accuracy of the whole procedure.

The representation using the series expansion approach also gives useful information regarding the behaviour of each stress and displacement component of each term included in the representation. For example, in Fig. 10 we have the angular behaviour of $\sigma_{\theta \theta}$ stress component around the " $90^{\circ}$ sector of $\left[0^{\circ}\right]$ CFRP - $270^{\circ}$ sector of adhesive" corner, where the dashed circle indicates the zero value, with tractions outside the circle and compressions inside it.

\section{Fig 10}

With this information we can observe the maximum tractions around the adhesiveCFRP interface at $90^{\circ}$ for term 1 while term 2 has the highest values inside the adhesive layer in the fourth quadrant around the $0^{\circ}$ interface.

With the present procedure, all configurations in Fig. 7 can be analyzed in detail, the values for each $K_{k}$ at each corner being comparable as they are associated to the same characteristic exponent. With this singularity characterization, the definition of an experimental test program has a higher added value as particular configurations can be found in which, for example, the value of $K_{1}$ (or $K_{2}$ ) changes in a controlled manner.

\section{CONCLUSIONS AND FUTURE DEVELOPMENTS}

In the present work a procedure for the calculation of Generalized Stress Intensity Factors (GSIFs) has been implemented based on a least squares procedure using the numerical results of BEM models.

Asymptotic stress and displacement representation by means of a series expansion allows the shape angular functions associated to each one of the terms of the series expansion to be determined. This gives a general validity to the representation, as the presence of an analyzed corner in another geometry configuration, or subjected to other load conditions, will only change the values of the associated GSIFs (the characteristic exponents and functions will be the same for the same local corner configuration). Thus, although the final stress or displacement fields will change, they will be obtained by means of the known shape angular functions multiplied by different weights (GSIFs).

The procedure described in this paper, together with a previous work by the same authors, Barroso et al. [25], has allowed the singularity characterization of several problems to be carried out. First, the method has proved to be robust and very accurate 
when applied to well known benchmark problems existing in the literature. Second, the procedure has been applied to study anisotropic multimaterial corners which typically appear in adhesively bonded lap joints between metals and composites. In particular the corners appearing in a double lap joint between aluminum and a CFRP laminate have been fully characterized, the local fields of displacements and stresses having been obtained.

The tool developed allows the definition of an oriented experimental test program to analyze the suitability of a GSIF based failure criterion in this kind of structural configuration. Different joints having locally the same corner configurations will allow the analysis of the role of the GSIFs in failure to be performed. In any case, it has to be stressed that GSIFs associated to different characteristic exponents have different dimensions and can therefore not be directly compared.

\section{ACKNOWLEDGEMENTS}

The authors gratefully acknowledge the financial support of the Spanish Ministry of Science and Technology (MAT2003-03315).

\section{REFERENCES}

[1] Sinclair, G. B. Stress singularities in classical elasticity - I: Removal, interpretation and analysis. ASME Appl. Mech. Rev. 2004 (57): 251-297.

[2] Joseph, P. F. and Zhang, N. Multiple root solutions, wedge paradoxes and singular stress states that are not variable-separable. Composite Science and Technology 1998 (58): 1839-1859.

[3] Gradin, A. and Groth, H. L. A fracture criterion for adhesive joints in terms of material induced singularities. Proc. of the Third International Conference on Num. Methods in Fracture Mechanics, Pineridge Press, Swansea, 1984.

[4] Groth, H. L. Stress singularities and fracture at interface corners in bonded joints. Int. J. of Adhesion and Adhesives 1988 (2): 107-113.

[5] Hattori, T. A stress-singularity-parameter approach for evaluating the adhesive strength of single lap joints. JSME International Journal, Series I, 1991 (34): 326-331. [6] Reedy, Jr., E. D. Intensity of the stress singularities at the interface corner between a bonded elastic and rigid layer. Engineering Fracture Mechanics 1990 (36): 575-583. [7] Reedy, Jr., E. D. and Guess, T. R. Butt tensile joint strength: interface corner stress intensity factor prediction. J. of Adhesion Sci. Tech. 1995(9): 237-251.

[8] Akisanya, A.R. On the singular stress field near the edge of bonded joints. Journal of Strain Analysis 1997 (32): 301-311.

[9] Yang, Y.Y. and Munz, D. Stress singularities in a dissimilar materials joint with edge tractions under mechanical and thermal loadings. International Journal of Solids and Structures 1997 (34): 1199-1216. 
[10] Lefebvre, D. R. and Dillard, D. A. A stress singularity approach for the predictions of fatigue crack initiation in adhesive bonds. Part 1: Theory. Journal of Adhesion 1997 (70): 119-138.

[11] Ishii, K., Imanaka, M., Nakayama, H. and Kodama, H. Evaluation of the fatigue strength of adhesively bonded CFRP/metal single and single-step double-lap joints. Composites Science and Technology 1999 (59): 1675-1683.

[12] Wang, C.H. and Rose, L.R.F. Compact solutions for the corner singularity in bonded lap joints. International Journal of Adhesion and Adhesives 2000 (20): 145154.

[13] Dunn, M. L., Hui, C. Y., Labossiere, P. E. W. and Lin, Y. Y. Small scale geometric and material features at geometric discontinuities and their role in fracture analysis. Int. J. of Fracture 2001 (110): 101-121.

[14] Penado, F. E. Singular intensity factors at bimaterial anisotropic interfaces. Composite Structures 2001 (52): 323-333.

[15] Lazzarin, P., Quaresimin, M. and Ferro, P. A two terms stress function approach to evaluate stress distributions in bonded joints of different geometry. Journal of Strain Analysis 2002 (37): 385-398.

[16] Leguillon, D. and Yosibash, Z. Crack onset at a v-notch. Influence of the notch tip radius. Int. J. of Fracture 2003 (122): 1-21.

[17] Quaresimin, M. and Ricotta, M. Stress intensity factors and strain energy release rates in single lap bonded joints in composite materials. Composite Science and Technology 2006 (66): 647-656.

[18] Dempsey, J.P. and Sinclair, G.B. On the stress singularities in the plane elasticity of the composite wedge. J. Elasticity 1979 (9): 373-391.

[19] Dempsey, J.P. and Sinclair, G.B. On the singular behaviour of a bi-material wedge. J. Elasticity 1981 (11): 317-327.

[20] Pageau, S.S., Joseph, P.F. and Biggers, JR., S.B. The order of stress singularities for bonded and disbonded three-material junctions. Int. J. Solids Struct. 1994 (31): 2979-2997.

[21] Ting, T.C.T. Stress singularities at the tip of interfaces in polycristals. Damage and Failure of Interfaces (Ed. by Rossmanith). Balkema, 1997, p. 75-82.

[22] Mantič, V. París, F. and Cañas, J. Stress singularities in 2D orthotropic corners. Int. J. Fracture 1997 (83): 67-90.

[23] Poonsawat, P., Wijeyewickrema, A.C. and Karasudhi, P. Singular stress fields of an anisotropic composite wedge with a frictional interface. ASCE 12th Eng. Mech. Conference, La Jolla, San Diego, CA, 1998, p. 578-581.

[24] Poonsawat, P., Wijeyewickrema, A.C. and Karasudhi, P. Singular stress fields of angle-ply and monoclinic bimaterial wedges. Int. J. Solids Struct. 2001 (38): 91-113. [25] Barroso, A., Mantič, V. and París, F. Singularity analysis of anisotropic multimaterial corners. Int. J. Fracture 2003(119): 1-23.

[26] Lekhnitskii, S. G. Some cases of the elastic equilibrium of a homogeneous cylinder with arbitrary anisotropy. Applied Mathematics and Mechanics (in Russian) 1938 (2): 345-367.

[27] Eshelby, J. D., Read, W. T. and Shockley, W. Anisotropic elasticity with applications to dislocation theory. Acta Metallurgica 1953 (1): 251-259. 
[28] Stroh, A. N. Dislocations and cracks in anisotropic elasticity. Philosophical Magazine 1958 (3): 625-646.

[29] Stroh, A. N. Steady state problems in anisotropic elasticity. Journal of Mathematics and Physics 1962 (41): 77-103.

[30] Ting, T.C.T. and Hwu, C. Sextic formalism in anisotropic elasticity for almost non-semisimple matrix N. Int. J. Solids and Structures 1988 (24): 65-76.

[31] Wang, Y.M. and Ting, T.C.T. The Stroh formalism for anisotropic materials that posses an almost extraordinary degenerate matrix N. International Journal of Solids and Structures 1997 (34): 401-413.

[32] Barroso, A., Mantič, V. and París, F. Computing stress singularities in transversely isotropic multimaterial corners by means of explicit expressions of the orthonormalized Stroh-eigenvectors. Engineering Fracture Mechanics 2007 (submitted for publication). [33] Tanuma, K. Surface impedance tensors of transversely isotropic materials. Q. J. Mech. Appl. Math. 1996 (49): 29-48.

[34] Yosibash, Z. and Szabó, B. A. A note on numerically computed eigenfunctions and generalized stress intensity factors associated with singular points. (Technical Note) Engineering Fracture Mechanics 1996 (54): 593-595.

[35] Pageau, S. P., Gadi, K. S., Biggers, Jr., S. B. y Joseph, P. F. Standardized complex and logarithmic eigensolutions for $\mathrm{n}$-material wedges and junctions. International Journal of Fracture 1996 (77): 51-76.

[36] Qian, Z.Q. and Akisanya, A.R. Wedge corner stress behaviour of bonded dissimilar materials. Theor. Appl. Fract. Mech. 1999 (32): 209-222.

[37] $\mathrm{Wu}$, K.C. Near-tip field and the associated path-independent integrals for anisotropic composite wedge. The Chinese J. of Mechanics 2001 (17): 21-28.

[38] Banks-Sills, L. and Sherer, A. A conservative integral for determining stress intensity factors of a bimaterial notch. Int. J. Fracture 2002 (115): 1-26.

[39] Cisilino, A.P. and Ortiz, J. Boundary element analysis of three-dimensional mixed-mode cracks via the interaction integral. Comput. Method Appl. M. 2005 (194): 935-956.

[40] Helsing, J. and Jonsson, A. On the computation of stress fields on polygonal domains with V-notches. Int. J. Numer. Meth. Eng. 2002 (53): 433-453.

[41] Helsing, J. and Jonsson, A. On the accuracy of benchmark tables and graphical results in the applied mechanics literature. ASME J. Appl. Mech, 2002 (69): 88-90.

[42] Graciani, E. Formulación e implementación del Método de los Elementos de Contorno para problemas axisimétricos con contacto. Aplicación a la caracterización de las interfase fibra matriz en materiales compuestos", PhD Thesis (in Spanish), University of Seville, 2006.

[43] Munz, D. and Yang, Y. Y. Stresses near the edge of bonded dissimilar materials described by two stress intensity factors. Int. J. Fracture 1993 (60): 169-177. [44] Ortiz, J.E., Mantič, V. and París, F. A domain-independent integral for computation of Stress Intensity Factors along three-dimensional crack front and edges by BEM. Int. J. Solids and Structures 2005 (43): 5593-5612. 


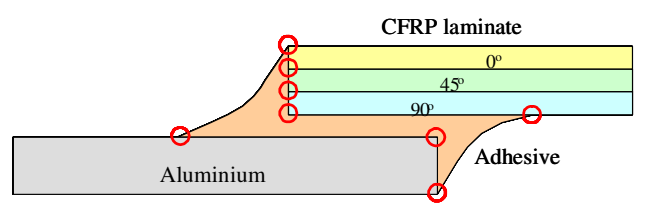

a)

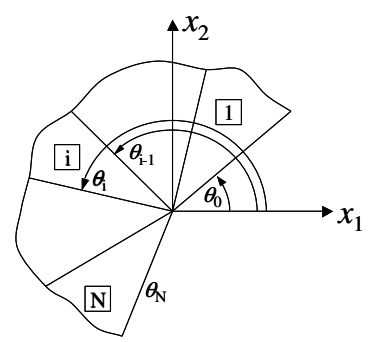

b)

Fig. 1 - Multimaterial corners in an Al-CFRP adhesively bonded single-lap joint.

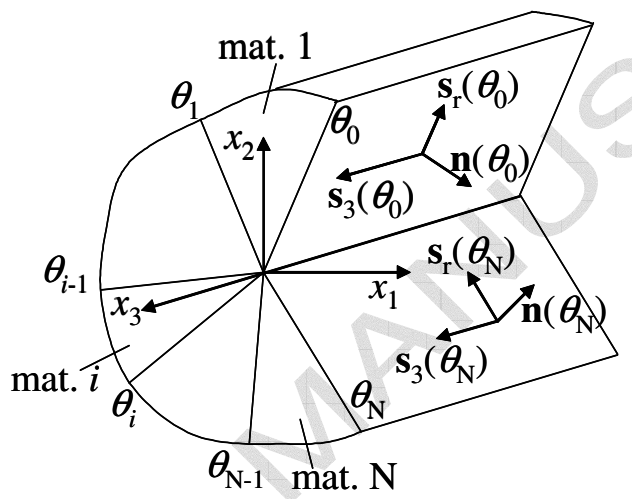

Fig. 2 - Unit outward normal for the definition of boundary conditions.

\begin{tabular}{|c|c|c|}
\hline \multirow[b]{2}{*}{ Boundary conditions } & \multicolumn{2}{|c|}{ Matrix definition } \\
\hline & $\mathbf{D}_{u}\left(\theta_{a}^{e}\right)$ & $\mathbf{D}_{\varphi}\left(\theta_{a}^{e}\right)$ \\
\hline Free & $\mathbf{0}$ & I \\
\hline Fixed & $\mathbf{I}$ & $\mathbf{0}$ \\
\hline Symmetry (only $u_{\theta}$ fixed) & {$\left[\mathbf{n}\left(\theta_{a}^{e}\right), \mathbf{0 , 0}\right]^{T}$} & $\left.\underset{T}{\left[\mathbf{0}, \mathbf{s}_{r}\right.}\left(\boldsymbol{\theta}_{a}^{e}\right), \mathbf{s}_{3}\left(\boldsymbol{\theta}_{a}^{e}\right)\right]$ \\
\hline $\begin{array}{l}\text { Antisymmetry (only } u_{\theta} \\
\text { allowed) }\end{array}$ & {$\left[\mathbf{s}_{r}\left(\theta_{a}^{e}\right), \mathbf{s}_{3}\left(\theta_{a}^{e}\right), \mathbf{0}\right]^{T}$} & {$\left[\mathbf{0 , 0}, \mathbf{n}\left(\theta_{a}^{e}\right)\right]^{T}$} \\
\hline Only $u_{r}$ fixed & {$\left[\mathbf{s}_{r}\left(\theta_{a}^{e}\right), \mathbf{0 , 0}\right]^{T}$} & {$\left[\mathbf{0}, \mathbf{n}\left(\theta_{a}^{e}\right), \mathbf{s}_{3}\left(\theta_{a}^{e}\right)\right]^{T}$} \\
\hline Only $u_{r}$ allowed & {$\left[\mathbf{n}\left(\theta_{a}^{e}\right), \mathbf{s}_{3}\left(\theta_{a}^{e}\right), \mathbf{0}\right]^{T}$} & {$\left[\mathbf{0 , 0}, \mathbf{s}_{r}\left(\theta_{a}^{e}\right)\right]^{T}$} \\
\hline Only $u_{3}$ fixed & {$\left[\mathbf{s}_{3}\left(\theta_{a}^{e}\right), \mathbf{0}, \mathbf{0}\right]^{T}$} & {$\left[\mathbf{0}, \mathbf{s}_{r}\left(\theta_{a}^{e}\right), \mathbf{n}\left(\theta_{a}^{e}\right)\right]^{T}$} \\
\hline Only $u_{3}$ allowed & {$\left[\mathbf{s}_{r}\left(\theta_{a}^{e}\right), \mathbf{n}\left(\theta_{a}^{e}\right), \mathbf{0}\right]^{T}$} & {$\left[\mathbf{0 , 0}, \mathbf{s}_{3}\left(\theta_{a}^{e}\right)\right]^{T}$} \\
\hline
\end{tabular}

Table 1.- Boundary condition matrices $\mathbf{D}_{u}\left(\theta_{a}^{e}\right)$ and $\mathbf{D}_{\varphi}\left(\theta_{a}^{e}\right)$, where $\theta_{a}^{e}(a=0,1)$ are defined as $\theta_{0}^{e}=\theta_{0}$ and $\theta_{1}^{e}=\theta_{N}$. 


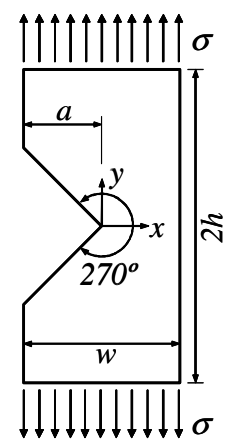

Fig. 3- Single-edge notched specimen.

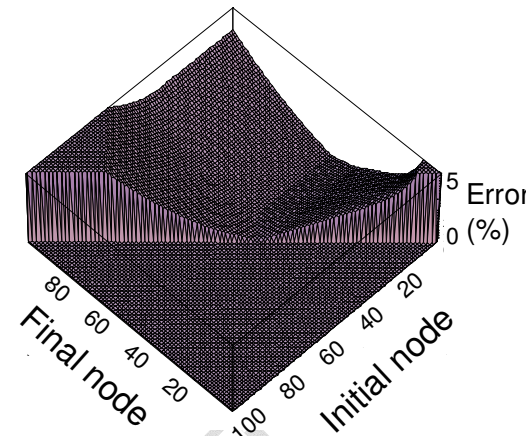

a)

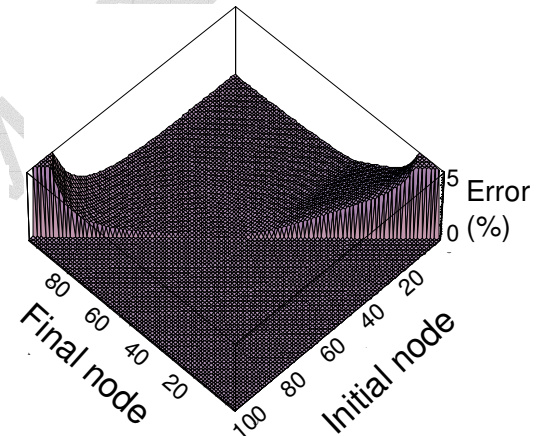

b)

Fig. 4- Relative errors (in \%) considering: a) 3 terms and b) 7 terms.
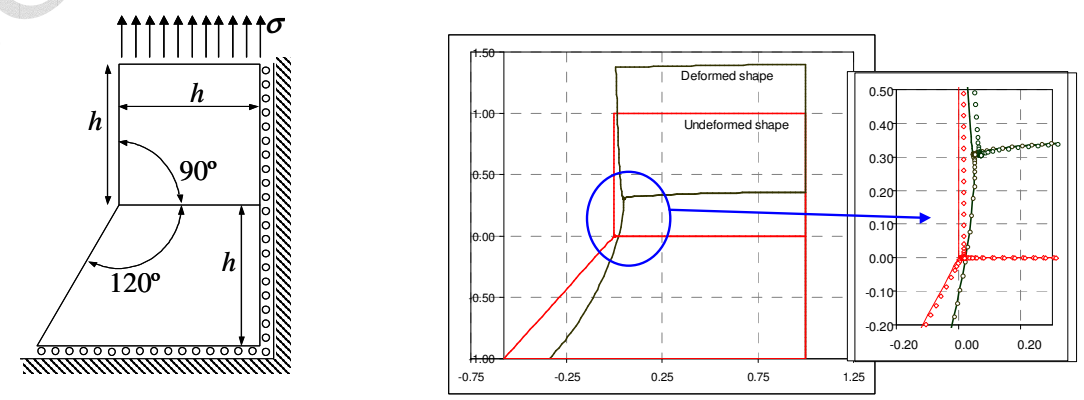

Fig. 5- Bimaterial corner configuration and BEM solution. 


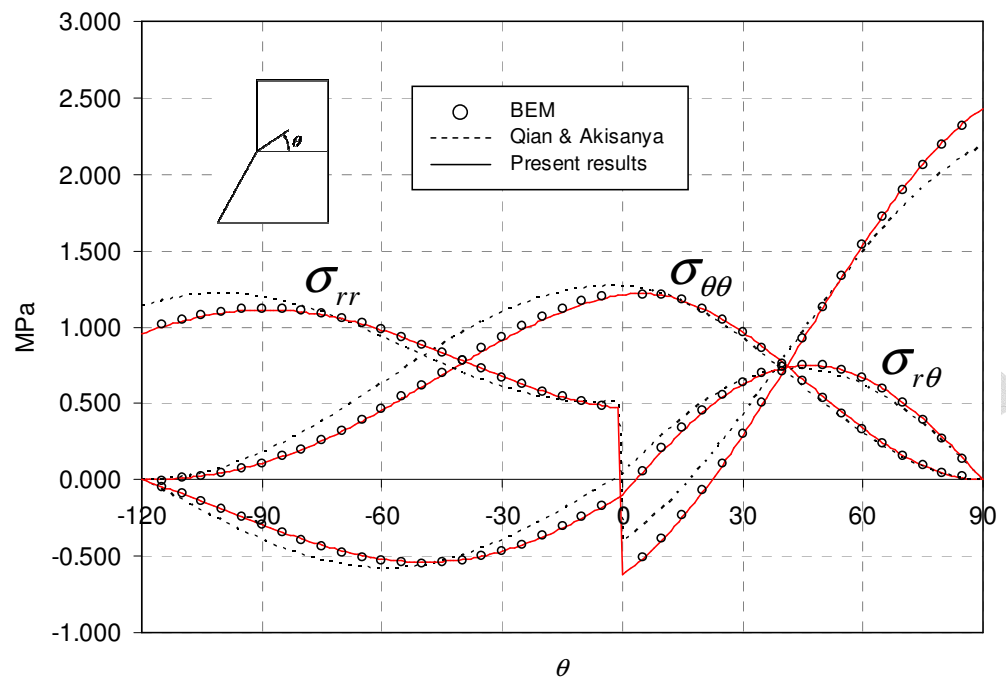

Fig. 6- Comparison of stresses in the bimaterial problem.

a)

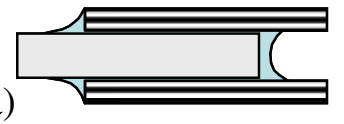

c)

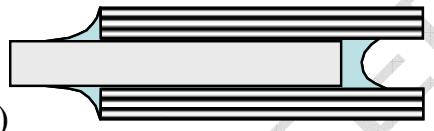

b)

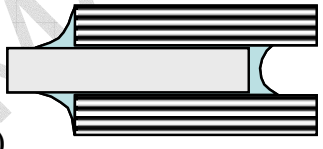

d)

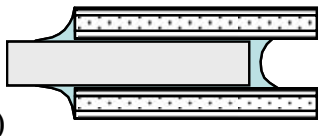

Fig. 7- a) Al (3.2)- $\left[0^{\circ}\right]_{8}(1.6) \mathrm{L}_{0}=12.5 \mathrm{~mm}$, b) Al (3.2)- $\left[0^{\circ}\right]_{16}(2.9) \mathrm{L}_{0}=12.5 \mathrm{~mm}$, c) Al (3.2) $\left.-\left[0^{\circ}\right]_{12}(2.2) \mathrm{L}_{0}=20 \mathrm{~mm}, \mathrm{~d}\right) \mathrm{Al}(3.2)-\left[0^{\circ}{ }_{2}, 9^{\circ}{ }_{2}\right]_{\mathrm{s}}(2.2) \mathrm{L}_{0}=12.5 \mathrm{~mm}$.

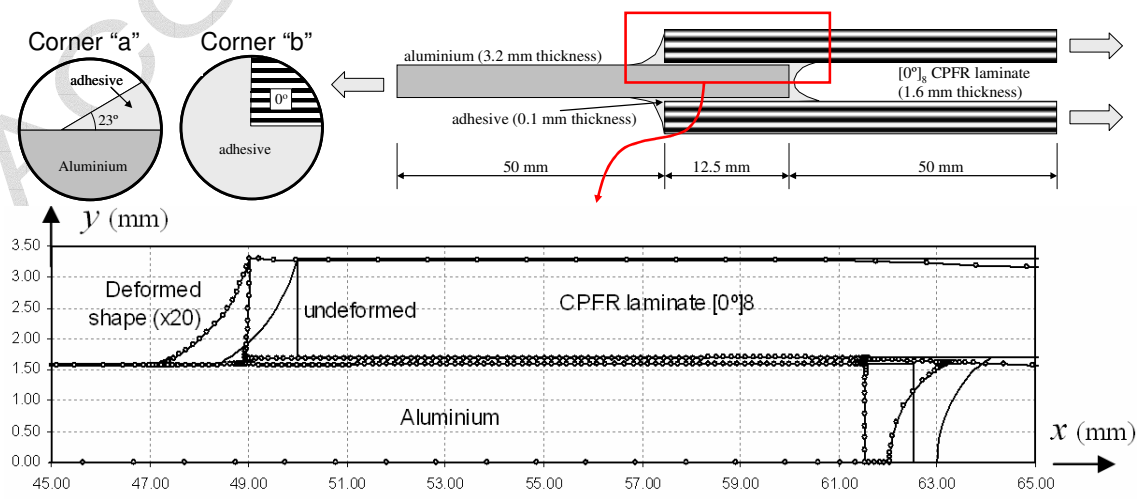

Fig. 8- Geometry (top) and detail of the BEM model (bottom). 


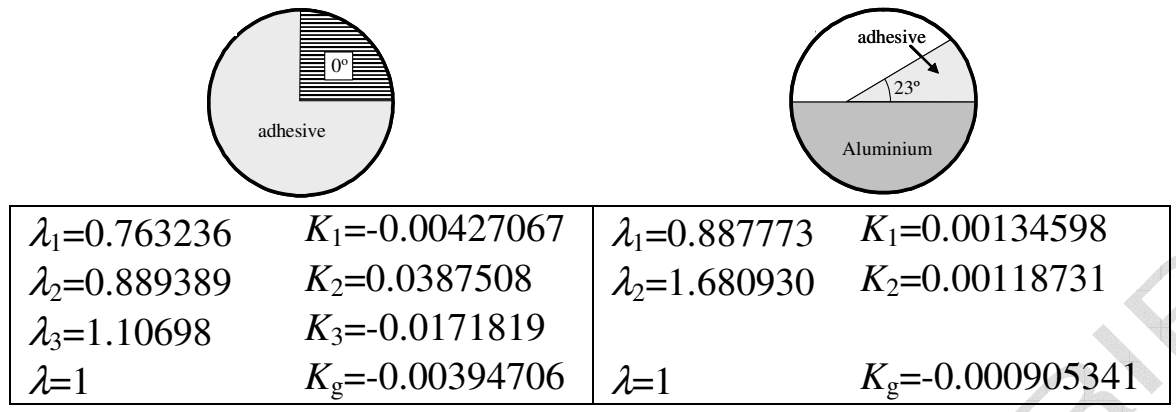

Table 2.- Values of $\lambda$ and $K$ for the analyzed corners.

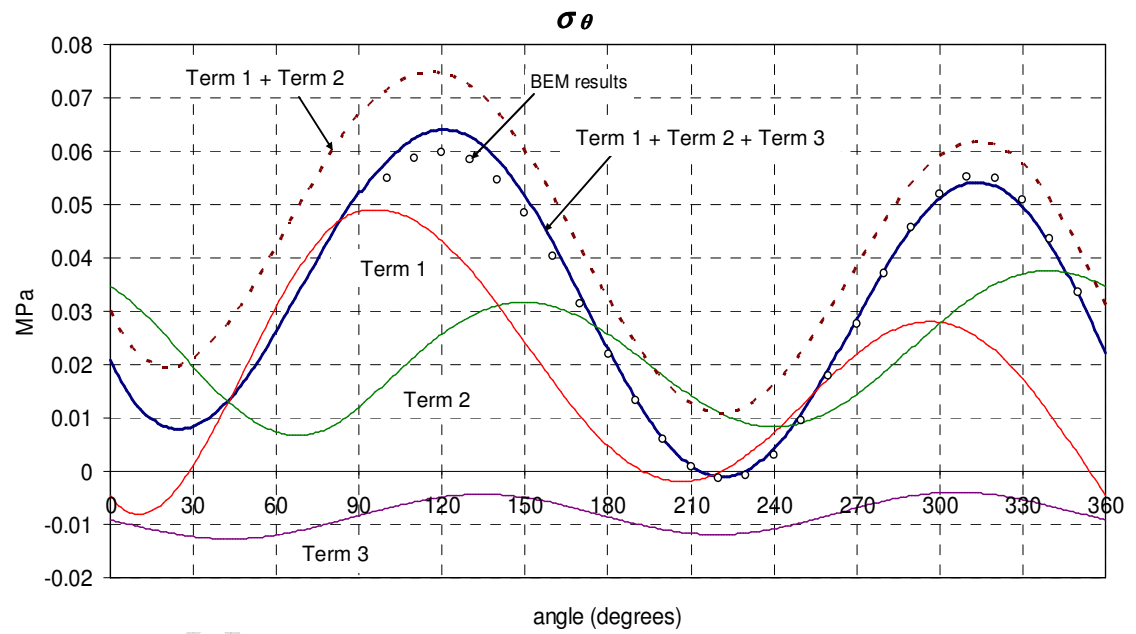

Fig. 9- $\sigma_{\theta \theta}$ in corner "b" $\left(90^{\circ}\right.$ wedge $\left[0^{\circ}\right]$ CFRP $-270^{\circ}$ wedge of adhesive).
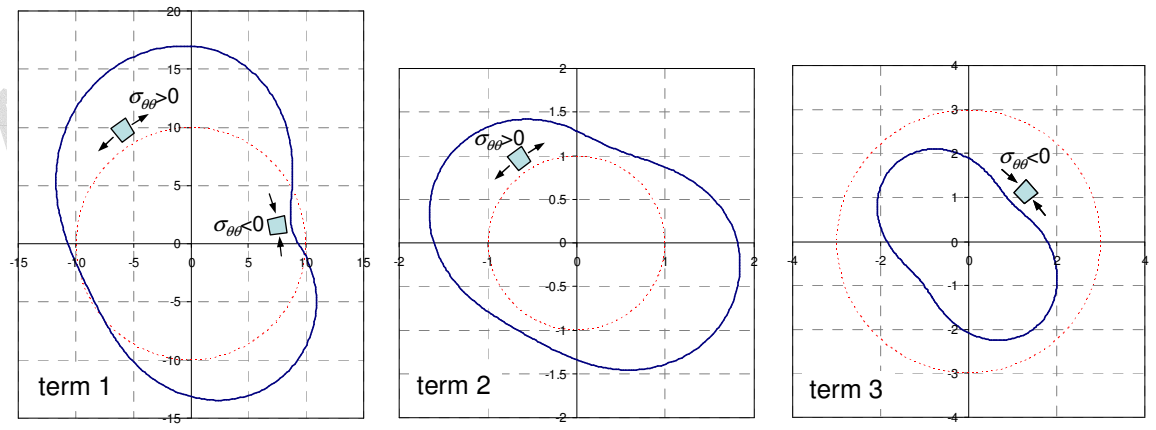

Fig. 10- $\sigma_{\theta \theta}$ for each term in the " $90^{\circ}\left[0^{\circ}\right]$ CFRP - $270^{\circ}$ adhesive" corner. 